\title{
TUBERCULOSE EXTRA GANGLIONNAIRE DE LA TÊTE ET DU COU
}

\author{
E. GASSAB, S. KEDOUS, A. BERKAOUI, N. SAYEH, K. HARRATHI, J. KOUBAA, A. GASSAB \\ SERVICE ORL ET CHIRURGIE CERVICO FACIALE. EPS FATTOUMA BOURGUIBA. MONASTIR
}

\begin{abstract}
RESUME
Les localisations ORL extra ganglionnaires de la tuberculose sont peu communes. La symptomatologie clinique est trompeuse, posant ainsi le problème de diagnostic différentiel avec la pathologie tumorale. Nous rapportons 12 cas de localisations extra ganglionnaires de tuberculose, colligés au service ORL de l'EPS Fattouma Bourguiba de Monastir entre 1995 et 2009. L'âge moyen de nos patients est de 38 ans. L'étude topographique a montré 3 localisations laryngées, 3 au niveau des glandes salivaires, 2 amygdaliennes, 2 rhinopharyngées, 2 rhino sinusiennes. Le diagnostic était anatomopathologique dans tous les cas. Tous nos patients ont reçu un traitement antituberculeux avec une bonne évolution. Mots-clés : Tuberculose, amygdale, rhinopharynx, larynx, glandes salivaires.
\end{abstract}

\section{SUMMARY}

Objective : ENT extra-nodal localisation of tuberculosis is an uncommon condition. Clinical symptomatology is misleading, so having the problem of differential diagnosis with tumoral disease. We report 12 cases of extra-nodal localisations of tuberculosis treated in ENT department of the Fattouma Bourguiba hospital of Monastir between 1995 and 2009 . The mean patients age is 38 years. Topographic analysis shows 3 laryngeal localisations, 3 on the salivary glands, 2 tonsillar, 2 on the nasopharynx and 2 rhinosinusal. The diagnosis was in all cases histo-pathological. All our patients received an antitubercular treatment with favorable evolution.

Keywords : Tuberculosis, larynx, nasopharynx, salivary glands, tonsil

\section{INTRODUCTION}

La tuberculose est une maladie infectieuse spécifique causée par une mycobactérie: le bacille de Koch. C'est une affection qui sévit encore dans notre pays à l'état endémique, la forme pulmonaire est de loin la plus fréquente ; les formes extra pulmonaires représentent $15 \%$; son incidence en ORL est une éventualité relativement rare, et si la forme ganglionnaire cervicale est la plus rencontrée d'autres localisations plus rares peuvent se voir. Le but de notre travail est de décrire ces différentes localisations, d'exposer les formes cliniques notamment les formes pseudo tumorales et d'insister sur l'intérêt de l'examen anatomopathologique dans cette pathologie.

\section{MATÉRIEL ET MÉTHODES}

Notre étude rétrospective a porté sur 12 cas de tuberculose extra ganglionnaire parmi 209 cas de tuberculose de la tête et du cou ( 5,7 \%) colligés au service ORL de l'EPS Fattouma Bourguiba de Monastir sur une période de 14 ans (1995-2009). Nos patients étaient repartis en 7 hommes et 5 femmes, la moyenne d'âge était de 38 ans ( de 4 à 84 ans), la vaccination par le BCG est retrouvée chez 7 patients, six malades sont d'origine rurale, le délai de consultation allait de 1 mois a 5 ans avec une moyenne de 12 mois.

Selon la répartition topographique notre série a comporté: - 3 localisations laryngée ; soit 25\%.

- 3 localisations au niveau des glandes salivaires; soit $25 \%$.

- 2 localisations nasopharyngiennes ; soit $16,6 \%$.
- 2 localisations rhino sinusiennes ; soit 16,6\%.

- 2 localisations amygdaliennes ; soit 16,6\%.

\section{RESULTATS}

Localisations laryngées :

Âge moyen de nos patients était de 51 ans, le sex-ratio 2 . Deux patients avaient une dysphonie isolée permanente évoluant depuis quelques mois sans $A E G$, sans fièvre. Une dysphagie haute aux solides sans dysphonie ni dyspnée avec notion de fièvre et amaigrissement ont été notés chez le troisième patient.

Une laryngoscopie directe a été pratiquée dans tous les cas, les aspects retrouvés sont:

- lésion kératosique du 1/3 antérieur de la corde vocale gauche.

- processus bourgeonnant irrégulier du bord libre épiglotte (Fig 1).

- aspect inflammatoire et boudiné de la corde vocale gauche.

Toutes les biopsies ont conclu à une tuberculose laryngée (Fig 2).

La radiographie du thorax était normale dans un cas. Des séquelles de tuberculose pulmonaire étaient notées chez un patient. Un aspect de tuberculose active avec BK positif dans les crachats a été relevé dans un cas. Tous nos patients ont eu 2 mois de quadrithérapie et 7 mois de bithérapie avec une bonne évolution ; le recul est de 18 mois en moyenne. 


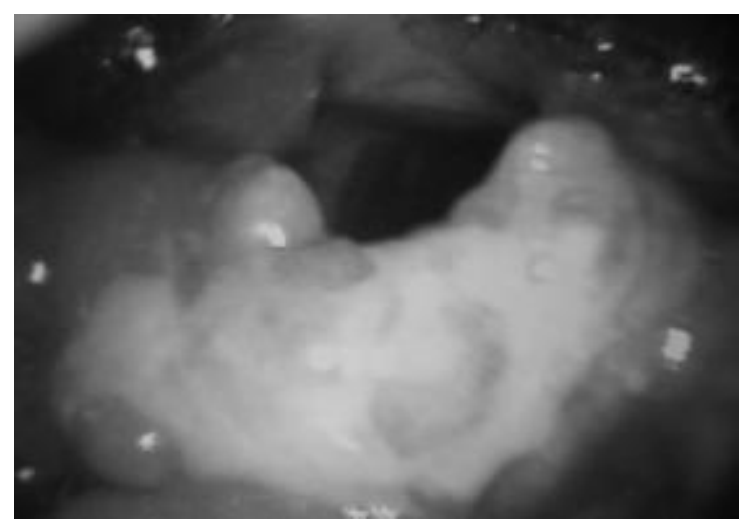

Fig. 1 : Vue endoscopique : Processus bourgeonnant irrégulier du bord libre de l'épiglotte étendu aux replis ary-épiglottiques .

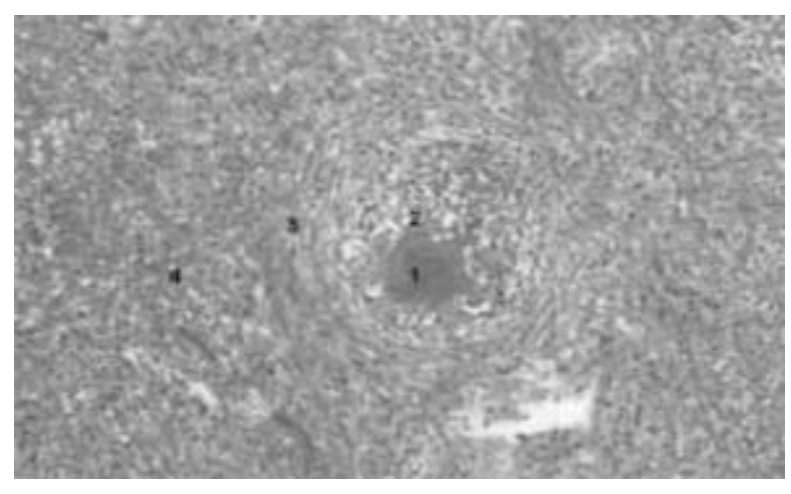

Fig. 2 : Nécrose granulomateuse. a HES $\times 10.1$ : cellule géante granulomateuse, 2 : plage de nécrose, 3 : couronne de lymphocytes, 4 : lymphocytes.

\section{Localisations amygdaliennes :}

Nos patients étaient âgés de 29 et 74 ans ; le sex-ratio est de 1. L'un consultait pour une odynophagie latéralisée à droite avec fièvre depuis 3 mois résistante au traitement médical avec découverte à l'examen d'une ulcération au niveau de l'amygdale droite recouverte d'un enduit blanchâtre dure à la palpation. L'autre patient consultait pour une tuméfaction laterocervicale depuis 6 mois spontanément fistulisée, on a trouvé une amygdale gauche congestive à l'examen clinique.

Une biopsie était pratiquée dans chacun des cas ; l'aspect retrouvé a l'examen anatomopathologique est celui d'amygdalite granulomateuse et nécrosante compatible avec une tuberculose (Fig 3). Une tuberculose pulmonaire associée est retrouvée chez un patient.

L'évolution sous traitement était favorable pour les deux localisations.

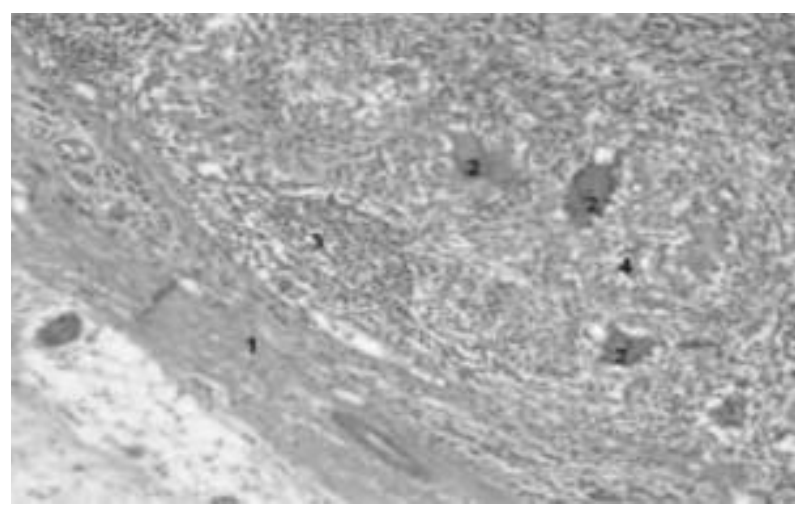

Fig. 3 : 1 : capsule glandulaire, 2 : cellules géantes multi nucléées, 3 : lymphocytes, 4 : plage de nécrose

Localisations au niveau des glandes salivaires : Il s'agissait de 2 femmes et un homme ; l'âge moyen était de 30 ans.

Dans les deux premiers cas le motif de consultation était une tuméfaction isolée de siège parotidien; notre examen avait mis en évidence une formation nodulaire bien circonscrite de la loge parotidienne, sans paralysie faciale, sans bombement para pharyngé, sans écoulement du Stenon.

Le troisième patient avait consulté pour une tuméfaction sous maxillaire droite sans notion de colique salivaire.

Une échographie a été pratiquée chez tous nos patients. L'examen anatomopathologique définitif a été fait sur une pièce de parotidectomie superficielle chez un patient, sur un nodule au dépend du prolongement inférieur de la glande parotide chez le deuxième et sur pièce de maxillectomie pour le troisième concluant dans tous les cas à des lésions granulomateuses et nécrosantes de nature tuberculeuse. Le traitement médical d'une durée totale de 9 mois était favorable pour tous les patients avec un recul de 6 mois.

\section{Localisations rhino sinusiennes :}

Nos deux patients étaient de sexe féminins ; de 37 et 40 ans ; le motif de consultation était une obstruction nasale chronique, des rhinorrhées purulentes et des épistaxis récidivantes. Une hyposmie était constatée chez une patiente.

L'endoscopie nasale a objectivé une muqueuse pituitaire inflammatoire granulomateuse saignante au contact dans 1 cas, une formation polyploïde blanchâtre provenant du méat moyen gauche dans l'autre. La VS était accélérée et I'IDR à la tuberculine était positive dans les 2 cas. La biopsie de la muqueuse sinusienne a retrouvé une tuberculose dans les 2 cas. La radiographie du thorax a objectivé une miliaire tuberculeuse, la recherche de BK dans les crachats était positive dans les deux cas. une TDM du massif facial a été pratiquée chez les deux malades montrant un comblement non spécifique des cavités naso 
sinusiennes sans lyse osseuse. L'évolution était favorable sous chimiothérapie anti-tuberculeuse pendant 8 mois, avec bonne tolérance et amélioration clinique.

\section{Localisations nasophayngiennes :}

Deux patients de sexe masculin âgé de 4 et 28 ans qui consultaient pour une tuméfaction latérocervicale isolée. Un examen systématique du cavum a montré un aspect sale sans formations tumorales. Une biopsie a été pratiquée concluant à la nature tuberculeuse de la muqueuse rhino pharyngée. L'adénectomie retrouve une tuberculose ganglionnaire associée. L'évolution sous traitement était favorable pour les deux localisations.

\section{DISCUSSION}

la tuberculose est un sujet d'actualité, d'après les données de l'OMS, 8 millions de nouveaux cas sont recensés chaque année dont 2 millions de décès.

La recrudescence actuelle de cette pathologie s'explique par l'épidémie du SIDA. [1].

La tuberculose extra ganglionnaire de la sphère ORL est une pathologie rare qui représente moins de $1 \%$ toute localisation confondue. [2]. Cependant cette pathologie reste sous estimée surtout dans un pays d'endémie comme la Tunisie car les localisations secondaires sont sous diagnostiquées du fait de la non systématisation de l'examen des voies aérodigestives supérieures devant toute atteinte tuberculeuse.

L'inoculation du site ORL par le bacille de Koch peut se faire par plusieurs voies $[3,4]$ :

- La voie hématogène à partir d'un site contaminé surtout pulmonaire.

- La voie lymphatique à partir d'une atteinte locorégionale cutanéomuqueuse.

- La voie canalaire par les expectorations bronchiques contenant les bacilles; c'est la diffusion ascendante.

- La voie directe à partir du milieu extérieur, ce mécanisme explique les formes primitives rares.

Ces différents mécanismes d'inoculation expliquent la diversité topographique lésionnelle et la possibilité des formes à localisations multiples.

L'atteinte laryngée représente l'essentiel de la tuberculose des VADS ; souvent associée à une tuberculose pulmonaire et peut être révélatrice de cette dernière dans $42 \%$ des cas [5,6]. Elle survient essentiellement chez le sujet de sexe masculin dont l'âge varie entre 40 et 60 ans. La dysphonie est le signe le plus fréquemment rencontré évoquant en premier lieu une pathologie néoplasique, d'autres manifestations non spécifiques sont décrites: l'odynophagie, le stridor, les algies cervicales, les toux productives et l'hémoptysie. Les aspects endoscopiques peuvent prendre plusieurs formes et prêtent à confusion dans les formes ulcèro bourgeonnantes et ulcèro infiltrantes avec la pathologie maligne ; les lésions prédominent à l'étage glottique, ce qui était le cas pour deux de nos patients, le troisième avait une formation bourgeonnante du bord libre de l'épiglotte.

C'est l'examen anatomopathologique avec recherche de BAAR qui permet le diagnostic de tuberculose laryngée et d'éliminer la pathologie néoplasique, tout en sachant que leurs association est possible [7].

L'atteinte oropharyngée reste dominée par l'atteinte amygdalienne observée dans $45 \%$ des cas [8]. L'atteinte pharyngée pure est exceptionnelle vu l'action nettoyante de la salive, la présence de germes saprophytes dans la cavité buccale ainsi que le PH local [9]. La symptomatologie est dominée par l'odynophagie et la dysphagie haute. A l'examen, on note généralement la présence d'une ulcération de la muqueuse oropharyngée ou une hypertrophie asymétrique d'une amygdale.

La présence d'adénopathies cervicales associées est notée dans $90 \%$ des cas, ceci est expliqué par la richesse du pharynx en tissu lymphoïde ; Une tuberculose pulmonaire évolutive associées aux localisations pharyngo laryngées est observée dans 30 à $96 \%$ des cas selon les séries [10].

Les atteintes tuberculeuses des glandes salivaires sont très rares [11,12], la localisation parotidienne est la plus fréquente décrite pour la première fois en 1894 par Von Stubenrauch, environ 200 cas ont été rapportés depuis cette date dans la littérature, elle atteint surtout l'adule jeune entre 30 et 50 ans ; l'atteinte est souvent secondaire et exceptionnellement primitive intéressant aussi bien le parenchyme parotidien que le tissu ganglionnaire péri glandulaire.

Deux voies de contamination glandulaire ont été décrites [13] :

- voie rétrograde à travers le canal de Stenon impliquant un foyer de contamination oropharyngé.

- voie hématogène ou lymphatique à point de départ pulmonaire dans le cadre d'une tuberculose disséminée.

Cliniquement, la tuberculose parotidienne se manifeste sous forme d'une tuméfaction de la glande unilatérale d'évolution progressive, diffuse ou nodulaire réalisant un syndrome pseudo tumoral. Une paralysie faciale périphérique, un trismus, et le caractère douloureux peuvent se voir mais orientent plus vers un processus malin. La recherche de notion de contage, de signes d'imprégnation et l'examen général peuvent orienter le diagnostic. La chirurgie constitue un moyen diagnostic de plus en plus abandonné par les auteurs vu la morbidité non négligeable et l'efficacité de traitement médical seul [14]. La cytoponction est peu pratiquée, elle fait courir au patient le risque d'atteinte du nerf facial et de fistulisation ; d'autant plus qu'elle n'a de valeur que si elle est positive. Certain auteurs exposent la possibilité de confirmation diagnostic par amplification génique après culture de prélèvements au niveau de l'orifice du canal de stenon ou à partir d'un broyat de cellules glandulaires [24].

La localisation rhinopharyngée de la tuberculose reste la 
plus retrouvée dans la littérature. Elle touche surtout les sujets jeunes ; l'âge moyen de nos patients est de 16 ans. Cliniquement, la symptomatologie est dominée par l'obstruction nasale, les épistaxis et les rhinorrhées. Des signes otologiques peuvent se voir à type d'otite séromuqueuse unilatérale. Des adénopathies cervicales associées sont observées dans 50 à $70 \%$ des cas [15,16], elles sont le plus souvent bilatérales de siège sous digastrique. L'aspect endoscopique le plus fréquent est celui d'une formation bourgeonnante ou ulcéro bourgeonnante essentiellement postéro supérieure. La TDM est non spécifique et peu orienter vers un processus malin du rhinopharynx d'où l'intérêt de pratiquer des biopsies systématiques du cavum.

Initialement décrite par Giovanni Morgani en 1761 sur des données d'autopsie ; la tuberculose nasosinusienne reste toutefois une entité rare estimée a $1 \%$ des tuberculoses de la tête et du cou [17]. La tuberculose nasale peut être classée en tuberculose nasale spontanée faisant suite à une tuberculose pulmonaire et la tuberculose nasale primaire qui survient sans atteinte pulmonaire préalable. Cette dernière entité reste rare vu l'action bactéricide des sécrétions nasales et la protection offerte par les mouvements ciliaires [18]. Les symptômes les plus fréquents sont l'obstruction nasale, la rhinorrhée, l'épistaxis comme constatés chez nos malades ainsi que la formation de croutes.

Les sites les plus intéressés sont la portion cartilagineuse du septum nasal et les cornets $[18,19]$, les lésions peuvent prendre la forme d'un tissu prolifératif granulomateux ou d'une ulcération pouvant prêter à confusion avec une granulomatose de Wegener ou une Sarcoïdose d'où l'intérêt de l'examen anatomopathologique et bactériologique.

D'autres localisations tuberculeuses on été décrites dans la littérature :

- la localisation thyroïdienne est très rare même dans les pays endémiques, décrite au 19eme siècle par Albers la tuberculose de la thyroïdes représente 0,1 à $0,4 \%$ de toutes les localisations. La rareté de cette affection s'explique par la bonne oxygénation du parenchyme thyroïdien ainsi que le rôle tuberculostatique des hormones thyroïdiennes. Le traitement est avant tout médical, devant une collection un drainage suffit, l'exérèse reste exceptionnelle.

- La tuberculose auriculaire reste une entité rare , représente $0,4 \%$ des otites moyennes selon Awan 1983 ; sa difficulté diagnostique réside dans le faite qu'elle se présente comme les autres otites moyennes suppuratives. La certitude de la tuberculose en ORL tout siège confondu est histologique et /ou bactériologique. L'aspect histologique est caractéristique devant la constation d'une nécrose caséeuse associée à un granulome épitheloide et gigantocéllulaire, l'absence de cette nécrose oriente vers les autres pathologies granulomateuses $[4,20,22$,
23]. Le fragment biopsique est mis en culture systématiquement sur milieu de Lobstein afin d'isoler le germe, typer l'espèce et réaliser un antibiogramme très utile dans les formes résistantes ou en cas d'antécédents de traitement anti tuberculeux ou sur un terrain particulier (HIV) [23]. D' autres procédés biologiques existent actuellement; plus rapides que la culture classique : culture en milieu liquide par dispositif MB-CHECK ou respiromètrie radiomètrie par méthode BACTEC. La recherche de l'acide tuberculo stéarique par méthode chromatographique et l'amplification génique PCR ( polymerase chain reaction) permettant une détection encore plus rapide en moins de 12 heures [23,24].

Le bilan de la maladie tuberculeuse comporte une étape $\mathrm{ORL}$ avec un examen complet et minutieux de la sphère $\mathrm{ORL}$ et une étape générale avec un bilan pleuropulmonaire. Le traitement de la tuberculose extra ganglionnaire de la tête et du cou se base essentiellement sur l'isoniaside, la rifampicine, la pyrazinamide et la streptomycine actuellement remplacée par l'ethambutol ; il se fait en association de 4 anti tuberculeux pendant 2 mois suivi d'un traitement double pendant 6 à 8 mois.

Dans la prise en charge de cette pathologie la chirurgie n'a qu'une place minime réservée aux formes pseudotumorales en vue d'une confirmation anatomopthologique, ailleurs elle est indiquée devant l'absence de régression des adénopathies sous anti tuberculeux ou en cas de complications tels qu'une fistule ou un abcès froid.

Le taux d'échec d'un traitement médical bien conduit est de l'ordre de 1\% [25], on doit alors évoquer une résistance du bacille de Koch aux anti-bacillaires d'où l'intérêt majeur de l'examen bactériologique afin d'identifier la souche impliquée et pratiquer un antibiogramme.

\section{CONCLUSION}

La tuberculose ORL extra ganglionnaire est une affection peu fréquente. Sa symptomatologie est non spécifique et son diagnostic est souvent posé à l'occasion d'une biopsie d'une lésion pseudotumorale.

Le traitement de la tuberculose est médical. II doit être bien conduit afin de minimiser la sélection des souches résistantes.

Le meilleur traitement reste préventif basé sur la vaccination au BCG et le dépistage précoce de l'atteinte tuberculeuse de la sphère ORL. 


\section{REFERENCES}

1) Chrétien $J$, Papillon $F$. La tuberculose et les mycobactérioses à l'ère du Sida Rev Prat. 1990;11;40(8):709-14

2) Wookyng Moon, Moon Hee Han, Jyun Chang et al. CT and MR imaging of head and neck. Tuberculosis Radiographies 1997; $17: 391-402$

3) Zanaret M, Dessi P. Tuberculose pharyngée. EMC ORL. 20-575-A-10,2p

4) El Amine M. N, El Alani, Essakalli H.L. La tuberculose du cavum. A propos de

2 cas. Les cahiers d'ORL $1993 ; 9$

5) D.Gallas, A.Coste, P.Bedbeder, R.Peynegre. Aspects actuels de la tuberculose laryngée à propos de 4 cas et revue de la literature. Ann Laryngol Rhinol Otol $1994 ; 111: 201-207$

6) S. Marfani et al. The larynx in pulmonary tuberculosis. J laryngol Otol 1985; $99: 201-203$

7) Essaadi M, Raji A, Detsouli M, Mokrim B, Kadiri F, Laraqui NZ, Touhami M, Chekkouri IA, Benchakroun Y. Rev Laryngol Otol Rhinol (Bord). 2001;122(2):125-8

8) Moulonguet L, Delguidice P, Chauvin JL. L'angine tuberculeuse : a propos d'un cas au Sénégal. . Ann Laryngol Rhinol Otol $1995 ; 112$ : 78-79.

9) Bath AP, O'Flynn P, Gibbin KP. Nasopharyngeal tuberculosis. J Laryngol Otol. 1992; 106(12):1079-80

10) Chobaut JC, Badet JM, Cantenot A, Lafon JC.J Fr Otorhinolaryngol Audiophonol Chir Maxillofac. 1982 ;31:157-68.

11) Franzen A, Franzen CK, Koegel K. Tuberculosis of the parotid gland: a rare differential diagnosis of parotid tumor. Laryngorhinootologie. 1997; 76:308-11. 12) Kontopoulou T, Fanourgiakis P, Samarkos M, Mintzias D, Peppas C, Rontogianni D, Vaidakis E. Tuberculosis of yhe parotid gland: case report and literature review. Med Mal Infect. $2004 ; 34: 488-90$.

13) M'barek C., Sethom A., Jhaider E. et al. Tuberculose primitive des glandes salivaires. Maghreb médical $2000 ; 20(352): 390-$

14) Benhammou A, El Ayoubi A, Benbouzid MA, Boulaich M, Essakali L, Kzadri M. Arch Pediatr. 2007;14(10):1206-9.
15) Waldron J, Van Hasselt CA, Skinner DW, Arnold M. Tuberculosis of the nasopharynx: clinicopthological features. Clin Otolaryngol Allied Sci. 1992;17(1):57-9.

16) Kh.Akkari, A.Sethom, H.Ouni, H.Brahem et coll. Tuberculose ORL extra ganglionnaire. J Tun ORL 2005;15:21-26.

17) Slutsker L, Castro KG, Ward JW, et al. Epidemiology of extrapulmonary tuberculosis among persons with AIDS in the United States. Clin Infect Dis 1993:16:513-8.

18) Goguen LA, Karmody CS. Nasal tuberculosis. Otolaryngol Head Neck Surg. 1995;113(1):131-5

19) Chodosh PL, Willis W. Tuberculosis of the upper respiratory tract. Laryngoscope 1970;80:679-696.

20) Bouzouita J, Rourou I, Abdelkafi M et al. La tuberculose des voies aerodigestives supérieures. J Tun ORL .1993; 4:2.

21) Beltaief $A$, Charfi .M , Skhiri I et al. La tuberculose des voies aéro-digestives supérieures. J Tun ORL. 2000;3:4.

22) Ennouri A, Maamouri M, Bouzouaïa N, Haji H, Bouzouita K, Ferjani $M$, Marrakchi H. Rev Laryngol Otol Rhinol . 1990;111(3):217-20

23) Kharoubi S. La tuberculose phayngée : Etude analytique à propos de 10 cas. Rev Laryngol Otol Rhinol . 1998;119(3):203-7.

24) Ikonomopoulos JA, Gorgoulis VG, Zacharatos PV, Manolis EN, Kanavaros $P$, Rassidakis A, Kittas C. Multiplex polymerase chain reaction for the detection of mycobacterial DNA in cases of tuberculosis and sarcoidosis.Mod Pathol. 1999;12(9):854-62.

25) Morris S, Bai GH, Suffys P. Molecular mechanisms of multiple drug resistance in clinical isolates of Mycobacterium tuberculosis. J Infect Dis 1995;171: 954-60. 